\title{
DESENVOLVIMENTO DE Rhapis excelsa (THUNBERG) HENRY EX. REHDER (PALMEIRA-RÁFIA): INFLUÊNCIA DA ALTURA DO RECIPIENTE NA FORMAÇÃO DE MUDAS
}

\author{
Development of Rhapis excelsa (Thunberg) Henry Ex. Rehder (Palm-Ráfia): influence of the \\ height of the recipient in the formation of seedlings
}

\author{
Petterson Baptista da Luz', Francismar Francisco Alves Aguiar², Armando Reis Tavares ${ }^{2}$, \\ Shoey Kanashiro ${ }^{2}$, Janaina Aguiar ${ }^{3}$, Thais Denise Rodrigues do Nascimento ${ }^{3}$
}

\begin{abstract}
RESUMO
Rhapis excelsa, originária do sul da China, é uma das palmeiras ornamentais mais cultivadas no mundo, principalmente em vaso. Objetivou-se com esta pesquisa investigar a influência da altura do vaso no desenvolvimento da espécie. Para tanto, mudas de dois anos de idade, apresentando $10 \mathrm{~cm}$ de altura e sistema radicular com $10 \mathrm{~cm}$ de comprimento, foram transplantadas para recipientes de PVC com $10 \mathrm{~cm}$ de diâmetro e 20,25,30,35 e $40 \mathrm{~cm}$ de altura, que se constituíram em tratamentos. O substrato utilizado foi uma mistura de casca de pinho, vermiculita e solo argiloso (2:1:1), adubado com $45 \mathrm{~g}$ de Osmocote (15:10:10). Os parâmetros avaliados foram: altura da planta, diâmetro do colo, número de folhas, número de perfilhos emitidos, avaliados aos 6,12 e 18 meses após a instalação do experimento. Aos 18 meses foram tomados também o peso da matéria seca da parte aérea e o peso da matéria seca das raízes. O delineamento experimental foi inteiramente casualizado, com cinco repetições com duas plantas. Houve diferença significativa para a variável altura de plantas, aos 6 meses, sendo que o recipiente de $40 \mathrm{~cm}$ de altura apresentou o pior resultado. Não houve diferença significativa entre as variáveis analisadas aos 12 meses de investigação. Já aos 18 meses, houve diferenças significativas entre os tratamentos para as variáveis altura da planta e diâmetro do colo, sendo o recipiente de $40 \mathrm{~cm}$ inferior aos demais em relação à altura da planta e o de $25 \mathrm{~cm}$ superior quanto ao diâmetro do colo. A utilização do recipiente com $25 \mathrm{~cm}$ de altura revelou-se interessante pela qualidade das plantas formadas e em relação a aspectos econômicos da produção.
\end{abstract}

Termos para indexação: Altura, recipiente, palmeira, mudas.

\begin{abstract}
Rhapis excelsa is one the most important ornamental palm cultivated in the world, mainly as vase plant, being indigenous to China. The aim of this research was to investigate the influence of vase height on the development of the species. Two years-old seedlings with $10 \mathrm{~cm}$ height and $10 \mathrm{~cm}$ root system length were transplanted to PVC containers with $10 \mathrm{~cm}$ diameter and 20, 25, 30, 35 and $40 \mathrm{~cm}$ height. The vase substratum was a mixture of pine bark, vermiculite and soil (2:1:1), fertilized with $45 \mathrm{~g}$ of Osmocote (15:10:10). The evaluated parameters were: plant height, stem based diameter, number of leaves and numbers of shoots, at 06,12 and 18 months after the beginning of the experiment. For 18 months plants, the foliar dry matter weight and the roots dry matter weight were also evaluated. The experimental design was completely randomized with five replicates and each experimental unit was composed of 2 plants. There was a significant difference for the parameter plants height at 6 months, and the $40 \mathrm{~cm}$ height container presented the worst results. There was no significant difference among the treatments at 12 months since transplantation. After 18 months, there were significant differences among the treatments for the parameters plant height and diameter of the lap, with plant height being lower for the $40 \mathrm{~cm}$ container than other treatments and the diameter of the lap being greater for $25 \mathrm{~cm}$ container. The use of $25 \mathrm{~cm}$ height containers was interesting because of the quality of the grown plants and in relation to the economical aspects of the production.
\end{abstract}

Index terms: Height, recipient, palm tree, seedlings.

(Recebido para publicação em 22 de setembro de 2004 e aprovado em 24 de agosto de 2005)

\section{INTRODUÇÃO}

As palmeiras são espécies vegetais bastante antigas e mesmo antes de Cristo já eram utilizadas de forma ornamental e na alimentação. A palmeira Rhapis excelsa pertencente à família Palmae (Arecaceae) que, compreende 198 gêneros e aproximadamente 2.650 espécies em todo o mundo (DOMINGUES, 1995).

Popularmente denominada palmeira senhora ou palmeira ráfia, acredita-se que esta espécie seja nativa do sul da China (BLOMBERY \& ROOD, 1982). Usualmente alcança 4,5 metros de altura e apresenta caules múltiplos, formando touceiras. Esses caules são cobertos com fibras marrons emaranhadas, as folhas são palmadas, divididas até a base em 5-9 segmentos irregulares (LORENZI, 1996). Possui um alto valor ornamental devido ao fato de ser pouco exigente em relação à luminosidade, tolerando para o seu desenvolvimento desde o pleno sol, quando adulta

\footnotetext{
'Doutorando, Departamento de Produção Vegetal - Universidade Estadual Paulista, Faculdade de Ciências Agrárias e Veterinárias - Campus de Jaboticabal - UNESP/FCAV - Via de Acesso Professor Paulo Donato Castellane, s/n - 14884-900 - Jaboticabal, SP - petterbaptista@yahoo.com.br ${ }^{2}$ Engenheiro Agronômo, Pesquisadores Científicos, Seção de Ornamentais, Instituto de Botânica - Secretaria do Meio Ambiente, SP -Cx. P. 4005 01061-970 - São Paulo, SP.

${ }^{3}$ Estagiária da Seção de Ornamentais do Instituto de Botânica, Secretaria do Meio Ambiente-SP, Cx. P. 4005 - 01061 -970 - São Paulo, SP.
} 
e baixa luminosidade como em escritórios, salas de estar, plantadas em vasos, valorizando muito o ambiente interno.

Sua multiplicação é realizada com o uso de sementes ou divisão de touceira, sendo mais difícil o primeiro processo, devido ao lento crescimento da planta.

O tipo de recipiente e suas dimensões exercem influência sobre a qualidade e custos de produção de mudas de espécie florestais (CARNEIRO, 1987).

$\mathrm{O}$ estudo das dimensões adequadas reveste-se de grande importância, pois recipientes com volume superior ao indicado provocam gastos desnecessários, elevam a área do viveiro, aumentam os custos de transporte, manutenção e distribuição das mudas no campo (CARNEIRO, 1995).

A reduzida altura dos recipientes na produção de mudas dificulta a drenagem, eleva a capacidade de retenção de água, podendo provocar encharcamento do substrato (MILKS et al., 1989). A saturação dos poros com água e conseqüente déficit de oxigênio prejudica o bom desenvolvimento do sistema radicular (GISLEROD, 1983).

Vários tipos e tamanhos de recipientes podem ser utilizados para a produção de mudas. Em trabalhos realizados por vários pesquisadores, verificaram que recipientes com maior volume de substrato apresentam uma tendência a produzir mudas mais vigorosas e de melhor qualidade (BESAGOITIA, 1980; GODOY \& GODOY JUNIOR, 1965; SILVEIRA et al., 1973; VIANA, 1964;).

Gomes et al. (1991) observaram que a seleção do tipo adequado de recipiente pode ser relevante, quando se obedece a um cronograma de plantio. Entretanto, as plantas com reduzida taxa de crescimento no viveiro, em virtude da restrição do crescimento radicular, causada pelo recipiente, se plantadas na mesma época, apresentam elevada taxa de crescimento e tendem com o decorrer do tempo, a se igualarem em altura às plantas produzidas em outros recipientes.

De acordo com Bõhm (1979), os recipientes devem possuir grande diâmetro ou grande altura para aumentar o volume para o crescimento das raízes, visto que as raízes devem ter adequado volume de substrato para enraizamento no sentido vertical, pois apresentam geotropismo positivo. Para Gomes et al. (1990), o diâmetro e altura dos recipientes utilizados na produção de mudas devem variar com as características de cada espécie e respectivo tempo de permanência no viveiro.

Objetivou-se com presente experimento investigar a influência da altura do recipiente no crescimento de mudas de $R$. excelsa.

\section{MATERIAL E MÉTODOS}

O experimento foi conduzido em estufa coberta com plástico de polietileno na Seção de Ornamentais do
Instituto de Botânica de São Paulo (coordenadas geográficas de 23³9'07" de latitude sul e 46³7'22" de longitude oeste de Greenwich, com altitude média de 798 metros acima do nível do mar). A pesquisa foi desenvolvida no período de julho de 2001 a dezembro de 2002.

Foram utilizados para a confecção dos recipientes 50 pedaços de tubo de PVC com $10 \mathrm{~cm}$ de diâmetro, cortados a $20,25,30,35,40 \mathrm{~cm}$ de altura e também 50 pratos de plástico. $\mathrm{O}$ prato foi colado na extremidade inferior dos recipientes para servir como base. Foram feitos quatro furos no tubo a $2 \mathrm{~cm}$ de altura para possibilitar a drenagem de água.

O substrato utilizado foi uma mistura composta por duas partes de casca de pinho, uma de vermiculita e uma de solo argiloso (2: 1: 1). Mediu-se o volume do substrato e da água até a capacidade de campo. As médias desses volumes constam na Tabela 1.

Utilizaram-se mudas com 2 anos de idade medindo em média $10 \mathrm{~cm}$ de altura, $10 \mathrm{~cm}$ de comprimento da raiz, 0,7 $\mathrm{cm}$ de diâmetro na altura do colo e 6,7 folhas. Estas mudas, anteriormente, eram mantidas em bandejas de isopor (tipo plantagil) com 128 células, em condições de estufa.

As mudas foram transplantadas com torrão de 10 cm de altura diretamente para os recipientes e mantidas dentro da estufa, recebendo irrigação diária por meio de aspersão, mantendo-as sempre na capacidade de campo.

As plantas receberam adubação com $45 \mathrm{~g}$ de osmocote (15: 10: 10) de liberação lenta, parcelada em seis doses de 7,5 g.

As variáveis de crescimento utilizadas na avaliação das respostas da planta foram: altura da planta, diâmetro do colo, número de folhas, número de perfilhos emitidos, avaliados aos 6, 12 e 18 meses após a instalação do ensaio. Aos 18 meses foram tomados também o peso da matéria seca da parte aérea (PMSA) e o peso da matéria seca das raízes (PMSR), calculando-se com isso a relação da biomassa da parte aérea e radicular (Tabela 2 ). O delineamento experimental utilizado foi inteiramente casualizado, com cinco repetições com duas plantas.

TABELA 1 - Altura dos recipientes, média dos volumes dos substratos e da água.

\begin{tabular}{ccc}
\hline $\begin{array}{c}\text { Altura dos } \\
\text { recipientes (cm) }\end{array}$ & $\begin{array}{c}\text { Vol. de } \\
\text { substrato (l) }\end{array}$ & Vol. de água (l) \\
\hline 20 & 1,3 & 0,45 \\
25 & 1,9 & 0,5 \\
30 & 2,1 & 0,6 \\
35 & 2,5 & 0,7 \\
40 & 2,9 & 0,85 \\
\hline
\end{tabular}

Ciênc. agrotec., Lavras, v. 30, n. 1, p. 31-34, jan./fev., 2006 
TABELA 2 - Relação de peso da matéria seca das raízes (PMSR) e peso de matéria seca da parte aérea (PMSA) de mudas de Rhapis excelsa 18 meses após a instalação do experimento.

\begin{tabular}{ccc}
\hline Recipientes & PMSR $($ g) & PMSA (g) \\
\hline $20 \mathrm{~cm}$ & $11,21 \mathrm{a}$ & $38,54 \mathrm{a}$ \\
$25 \mathrm{~cm}$ & $11,96 \mathrm{a}$ & $37,72 \mathrm{a}$ \\
$30 \mathrm{~cm}$ & $12,03 \mathrm{a}$ & $39,63 \mathrm{a}$ \\
$35 \mathrm{~cm}$ & $14,50 \mathrm{a}$ & $45,45 \mathrm{a}$ \\
$40 \mathrm{~cm}$ & $12,43 \mathrm{a}$ & $39,82 \mathrm{a}$ \\
\hline
\end{tabular}

\section{RESULTADOS E DISCUSSÃO}

Não houve diferença significativa para o peso da matéria seca da parte aérea (PMSA) e o peso da matéria seca das raízes (PMSR), (Tabela 2).

De acordo com a análise de variância, houve diferença significativa para a variável altura de plantas, aos 6 meses, sendo que o recipiente de $40 \mathrm{~cm}$ de altura apresentou a menor média para os parâmetros estudados, quando comparado com os outros recipientes. Não houve diferença significativa entre as variáveis analisadas aos 12 meses de investigação. Já aos 18 meses, houve diferenças significativas entre os tratamentos para as variáveis altura da planta e diâmetro do colo, sendo que o recipiente de $40 \mathrm{~cm}$ mostrou-se inferior aos demais em relação a altura da planta e o de $25 \mathrm{~cm}$ mostrou-se superior quanto ao diâmetro do colo (Tabela 3).

Queiroz et al. (2001) relatam que recipientes de tamanho médio $(17 \times 22 \mathrm{~cm})$ e grande $(20 \times 27 \mathrm{~cm})$ são mais apropriados para o desenvolvimento de mudas de açaí.

Em cultivares de zínia, o tamanho do vaso não influenciou significativamente alguns parâmetros importantes na avaliação da qualidade das plantas, como o diâmetro de capítulos e altura da parte aérea (PINTO et al., 2003).

Puchalski \& Kämpf (1999) verificaram que o percentual de enraizamento, número e comprimento de raízes foram incrementados pela redução gradativa na altura do recipiente em mudas de Hibiscus rosa-sinensis $\mathrm{L}$.

A reduzida altura dos recipientes na produção de mudas em "plugs" dificulta a drenagem, eleva a capacidade de retenção de água, podendo provocar encharcamento do substrato (MILKS et al., 1989). Isso pode explicar o menor crescimento das mudas de R. excelsa nos vasos de $40 \mathrm{~cm}$, devido ao fato deste reter menos água.

Melo (1999) trabalhando com dois tamanhos de tubetes $(50 \mathrm{~mL}$ e $120 \mathrm{~mL})$ na produção de mudas de cafeeiro, não observou diferenças significativas na altura de plantas, permitindo a obtenção de mudas com padrão semelhante.

TABELA 3 - Valores médios de altura e diâmetro de mudas de Rhapis excelsa aos 6 e 18 meses após a instalação do experimento.

\begin{tabular}{ccccc}
\hline Tempo de Coleta & \multicolumn{2}{c}{ 6 meses } & \multicolumn{2}{c}{18 meses } \\
\hline Recipientes & Altura $(\mathbf{c m})$ & Diâmetro $(\mathbf{c m})$ & Altura $(\mathbf{c m})$ & Diâmetro $(\mathbf{c m})$ \\
\hline $20 \mathrm{~cm}$ & $15,40 \mathrm{a}$ & $1,37 \mathrm{a}$ & $37,00 \mathrm{a}$ & $2,02 \mathrm{c}$ \\
$25 \mathrm{~cm}$ & $13,80 \mathrm{a}$ & $1,27 \mathrm{a}$ & $36,10 \mathrm{a}$ & $2,42 \mathrm{a}$ \\
$30 \mathrm{~cm}$ & $13,80 \mathrm{a}$ & $1,37 \mathrm{a}$ & $36,00 \mathrm{a}$ & $1,95 \mathrm{c}$ \\
$35 \mathrm{~cm}$ & $14,40 \mathrm{a}$ & $1,33 \mathrm{a}$ & $38,65 \mathrm{a}$ & $2,12 \mathrm{~b}$ \\
$40 \mathrm{~cm}$ & $12,60 \mathrm{~b}$ & $1,15 \mathrm{a}$ & $31,85 \mathrm{~b}$ & $2,08 \mathrm{~b}$ \\
$\mathrm{CV}$ & $8,44 \%$ & $9,23 \%$ & $9,19 \%$ & $4,65 \%$ \\
\hline
\end{tabular}

Médias seguidas da mesma letra não diferem entre si ao nível de 5\% de probabilidade pelo teste de Scott-Knott. 


\section{CONCLUSÃO}

A utilização do recipiente com $25 \mathrm{~cm}$ de altura revelou-se interessante pela qualidade das plantas formadas e em relação a aspectos econômicos da produção, e existe uma correlação direta entre a biomassa radicular e a biomassa da parte aérea em $R$. excelsa, na qual observamos que quanto maior o PSMR maior o PSMA.

\section{REFERÊNCIAS BIBLIOGRÁFICAS}

BESAGOITIA, M. C. R. Efecto del tamaño de la bolsa en el desarrollo del cafeto cultivares 'Bourbon' y 'Pacas' en vivero. Resúmenes de Investigaciones en Café, Nueva San Salvador, v. 3, p. 71-72, 1980.

BLOMBERY, A.; ROOD, T. Palms: an informative practical guide to palms of the world: their cultivation, care and landscape use. North Ryde: Angus; London: Robertson, 1982. $201 \mathrm{p}$.

BÖHM, W. Methods of studying root system. Berlim: Springer Verlag, 1979. $188 \mathrm{p}$.

CARNEIRO, J. G. A. Influência de recipientes e de estações de semeadura sobre o comportamento do sistema radicular e dos parâmetros morfológicos de mudas de Pinus taeda e Pinus elliottii L. Curitiba: UFPR, 1987. 81 p.

CARNEIRO, J. G. A. Produção e controle de qualidade de mudas florestais. Curitiba: UFPR-FUPEF, 1995. 451 p.

DOMINGUES, R. C. Mudas florestais. Revista dos Amantes da Natureza, [S.1.], v. 89, n. 5, p. 14-18, 1995.

GISLEROD, H. R. Physical conditions of propagation media and their influence on the rooting of cuttings: III. the effect of air content and temperature in different propagation media on the rooting of cuttings. Plant and Soil, The Hague, v. 75, p. 1-14, 1983.

GODOY, O. P.; GODOY JÚNIOR, C. Influência da adubação no desenvolvimento de mudas de café. Revista de Agricultura, Piracicaba, v. 40, n. 3, p. 125-129, set. 1965.

GOMES, J. M.; COUTO, L.; BORGES, R. C. G.; FONSECA, E. P. Efeitos de diferentes substratos na produção de mudas de Eucalyptus grandis Hill ex Maidem, Win-strip. Árvore, Viçosa, v. 15, n. 1, p. 35-42, 1991.

GOMES, J. M.; COUTO, L.; BORGES, R. C. G.; FREITAS, S. C. Influência do tamanho da embalagem plástica na produção de mudas de Ipê, Copaíba e Angico Vermelho. Árvore, Viçosa, v. 14, n. 1, p. 26-34, 1990.

LORENZI, H. Palmeiras no Brasil: nativas e exóticas. Nova Odessa: Plantarum, 1996. 320 p.

MELO, B. de. Estudos sobre produção de mudas de (Coffea arabica L.) em tubetes. 1999. 119 p. Tese (Doutorado em Fitotecnia) - Universidade Federal de Lavras, Lavras, 1999.

MILKS, R. R.; FONTENO, W. C.; LARSON, R. A. Hidrology of horticultural substrates: II. predicting physical properties of substrate in containers. Journal American Society Horticulture Science, Alexandria, v. 144, n. 1, p. 52-56, 1989.

PINTO, A. C. R.; RODRIGUES, T. J. D.; LEITE, I. C.; BARBOSA, J. C. Efeito de tamanho de vaso e sistema de condução no desenvolvimento e qualidade de cultivares de ziniz. Revista Brasileira de Horticultura Ornamental, Campinas, v. 9, n. 1, p. 53-62, 2003.

PUCHALSKI, L. E. A.; KÄMPF, A. N. Efeito da altura do recipiente sobre a produção de mudas de Hibiscus rosasinensis L. em plugs. In: ENCONTRO NACIONAL SOBRE SUBSTRATOS PARA PLANTAS (ENSUB), 1., 1999, Porto Alegre, RS. Anais... Porto Alegre: [s.n.], 1999. p. 209-215.

QUEIROZ, J. A. L.; MELÉN JÚNIOR, N. J. Efeito do tamanho do recipiente sobre o desenvolvimento de mudas de açaí. (Euterpe oleracea Mart.). Revista Brasileira de Fruticultura, Jaboticabal, v. 23, n. 2, p. 460-462, 2001.

SILVEIRA, A. J. da; SANTANA, A. P.; PEREIRA, M. P. Efeito do tamanho do saco plástico e do método de semeadura no desenvolvimento de mudas de café. Seiva, Viçosa, v. 33, n. 77, p. 14-18, 1973.

VIANA, A. C. C. Desenvolvimento de mudas de café em bolsas de polietileno. Ciência e Cultura, São Paulo, v. 16, n. 2, p. 142-143, 1964.

Ciênc. agrotec., Lavras, v. 30, n. 1, p. 31-34, jan./fev., 2006 